\title{
Quality of Service Issues for Reinforcement Learning Based Routing Algorithm for Ad-Hoc Networks
}

\author{
Shrirang Ambaji Kulkarni ${ }^{1}$ and G. Raghavendra $\mathrm{RaO}^{2}$ \\ ${ }^{1}$ Gogte Institute of Technology, Karnataka, India \\ ${ }^{2}$ National Institute of Engineering, Karnataka, India
}

\begin{abstract}
Mobile ad-hoc networks are dynamic networks which are decentralized and autonomous in nature. Many routing algorithms have been proposed for these dynamic networks. It is an important problem to model Quality of Service requirements on these types of algorithms which traditionally have certain limitations. To model this scenario we have considered a reinforcement learning algorithm SAMPLE. SAMPLE promises to deal effectively with congestion and under high traffic load. As it is natural for ad-hoc networks to move in groups, we have considered the various group mobility models. The Pursue Mobility Model with its superior mobility metrics exhibits better performance. At the data link layer we have considered IEEE 802.11e, a MAC layer which has provisions to support QoS. As mobile ad-hoc networks are constrained by resources like energy and bandwidth, it is imperative for them to cooperate in a reasonably selfish manner. Thus, in this paper we propose cooperation with a moderately punishing algorithm based on game theory. The proposed algorithm in synchronization with SAMPLE yields better results on IEEE 802.11e.
\end{abstract}

Keywords: Quality of Service, reinforcement learning based routing protocol, group mobility models, routing metrics, game theory

\section{Introduction}

IEEE 802.11e provides Enhanced Distributed Coordination Function (EDCF) to support Quality of Service (QoS) [1]. The traditional IEEE 802.11 Medium Access Control (MAC) is based on Distributed Coordination Function (DCF) which is based on Carrier Sense Multiple Access with Collision Avoidance (CSMA/CA). SAMPLE [2] a reinforcement learning based routing algorithm models its reward policy based on the number of attempts for successful unicast transmission on IEEE 802.11. The DCF is the only mechanism by which 802.11 assures some form of QoS. Thus we extend the study of SAMPLE to deal with better QoS facilities provided by 802.11 e. Group mobility models exhibit collaborative behavior and naturally complement the functioning of Mobile Ad-Hoc Networks (MANETS). The mobility characteristics like speed, direction and correlation with nodes have a profound effect on routing protocols [3]. These characteristics of mobility models are studied with various mobility metrics to analyze their impact on reinforcement learning based routing algorithm SAMPLE. The goal of this work is to study the effects of EDCF on the reward model of SAMPLE with different priority traffic on varying group mobility models. As ad-hoc networks do not have a centralized mechanism for communication, they must agree upon a model of cooperation. This model of cooperation is further compounded by the presence of severe resource constraints. If the mobile nodes act selfishly, they might not utilize their transmission power to transmit other node packets. We model this scenario as a noncooperative game theory. A moderately punishing game theoretic algorithm is proposed and the effects of SAMPLE on different MAC layers like IEEE 802.11e and IEEE 802.11 is studied.

The rest of the paper is organized as follows. In Section 2 we discuss related work. Section 3 briefly discusses 802.11 and 802.11 e. Section 4 presents the SAMPLE routing protocol. Section 5 discusses the Group Mobility Models. Section 6 discusses the metrics, simulation environment and results. Section 7 discusses the proposed moderately punishing algorithm. Section 8 concludes the work. 


\section{Related Work}

J. Dowling et al. [2] implemented and analyzed a reinforcement learning algorithm SAMPLE on IEEE 802.11. They considered the Random Waypoint Mobility Model which modeled node movement in random fashion. SAMPLE exhibited better performance as compared to Ad-Hoc On Demand Distance Vector (AODV) and Dynamic Source Routing (DSR in terms of congestion handling capabilities. Thus, we considered SAMPLE for modeling QoS related performances. Perkins and Belding [19] proposed extensions to the routing protocol AODV. These extensions were usually associated with messages used during the route discovery. Their proposed draft explored as to how the service guarantees were met in AODV. Loscri [20] et al. considered AdHoc On-demand Multipath Distance Vector Routing (AOMDV) for evaluation on IEEE 802.11 and Evolutionary-TDMA (ETDMA). The performance results showed a better output in terms of throughput and average end-to-end delay of multipath (AOMDV) over E-TDMA. C. T. Calafate et al. [14] proposed a novel QoS architecture that was able to support applications with the bandwidth, delay and jitter requirements in MANET environments. They relied on IEEE 802.11e technology to offer soft QoS support to MANETs. By considering H.264/AVC reference software they obtained good video PSNR.

Puschita and Tudor [5] evaluated a layered approach for QoS to offer the possibility of selection of certain configuration according to network capabilities and applications availability. They observed that traffic stream efficiency increases in case of using EDCF as compared to DCF. Also high priority traffic streams allow a high data transfer rate. EDCF enables traffic balancing offering transmission opportunities to all nodes in the network. C. T. Calafate et al. [15] observed considerable improvements in terms of throughput and normalized routing overhead to the increased routing responsiveness due to IEEE 802.11e. They observed that employing IEEE 802.11e improved performance of multimedia traffic and routing efficiency.

L. Khouki and S. Cherkaoui [21] proposed a new intelligent cross-layer QoS support for wireless mobile ad-hoc networks known as FuzzyQoS. FuzzyQoS exploited fuzzy logic for traffic regulation and the control of congestion for both real-time multimedia (audio/video) services and non-real-time traffic services. They evaluated the performance of FuzzyQoS using the ns-2 simulator under varying mobility, traffic, and channel conditions. In terms of traffic scalability, the simulations showed a gain in terms of average end-to-end delay by about $74 \%-$ $92 \%$ and $49 \%$ in comparison to, the original IEEE 802.11 wireless networks A. Munaretto and M. Fonseca [22] proposed the QOLSR protocol which added the quality parameters to the standard OLSR. QOLSR was implemented on the OPNET simulator. The bandwidth measurements were calculated using IEEE $802.11 \mathrm{~b}$ as the medium access control protocol. The three QOLSR variants achieved better performance as compared with the standard OLSR protocol. T. Bheemarjuna Reddy et al. [23] surveyed the issues and challenges in providing QoS or adhoc wireless networks. They observed that the characteristics of ad-hoc wireless networks such as of not having central coordination, mobility of hosts, and limited availability of resources made QoS provisioning very challenging.

M. A. Igartua et al. [24] proposed g-MMDSR a game theoretic-Multipath Multimedia Dynamic Source Routing, which was a cross-layer multipath routing protocol and utilized game theory to achieve a dynamic selection of the forwarding paths. The proposed g-MMDSR was an extension to DSR (Dynamic Source Routing). They conducted simulations in the open source network simulator ns-2 (v2.27). Simulation results showed the benefits of their proposed work under high interfering traffic and mobility of the nodes. D. Levin [25] used game theory to examine existing internal incentive mechanisms for wireless networks based on IEEE 802.11. He showed that isolation does not always ensure cooperation and, on the other hand, jamming, though seemingly malicious, is a viable means by which to enforce cooperation of each node in the system. He observed that the price of high jamming can result in significant loss of efficiency. E. Altman et al. [26] proposed a simple punishing mechanism for nodes in a non-cooperative ad-hoc environment. The advantage of the proposed strategy was that it resulted in a less "aggressive" equilibrium on the contrary to a degenerative scenario whereby a node either forwards all the requested traffic or does not forward any of the requests. This led to the motivation of the study whereby the initial negative loop formed due to the punishment 
of neighbors is avoided and the cooperative behavior of node is considered dynamically and brought back into the mainstream operations of ad-hoc network.

\section{IEEE 802.11 versus IEEE 802.11e}

802.11 MAC uses DCF mode to access the channel for ad-hoc networks [4]. A basic form of QoS is achieved in 802.11 through collision avoidance [5]. The orientation of QoS support using DCF in IEEE 802.11 is via no service differentiation, a contention based media access along with a packet scheduler based on first in first out (FIFO) strategy. This imposes constraint on QoS applications which require priority packet scheduling. $802.11 \mathrm{e}$ on the other hand relies on EDCF to provide differentiated QoS services supporting around eight distinct traffic classes. EDCF supports per-class QoS services with contention based media access along with priority packet scheduling. Thus EDCF realizes the desired QoS services. The packet scheduler arbitrates virtual collisions by delivering a transmission opportunity (TxOP) to the highest priority traffic [6].

\section{SAMPLE}

SAMPLE [2] is a collaborative reinforcement (CRL) routing algorithm for MANETs. It heavily draws inspiration from prioritized sweeping learning technique [7]. Prioritized sweeping converges to optimal solutions by following interesting information in a given solution space. SAMPLE is based on a reward policy which approximates the number of transmissions needed to transmit a packet on IEEE 802.11. Approximately seven tries are required before a packet transmission failure occurs. Thus the rewards are set to -1 and -7 for success and failure of unicast transmissions. The model-based reinforcement learning algorithm employed by SAMPLE is specified in Equation 1:

$$
\begin{aligned}
& Q_{i}(s, a)=R(s, a)+\sum_{s^{\prime} \in s i} P_{i}\left(s^{\prime} / s, a\right) \\
& \cdot\left(D_{i}\left(s^{\prime} / s, a\right)+\operatorname{Decay}\left(V_{j}\left(s^{\prime}\right)\right)\right),
\end{aligned}
$$

where $a \in A_{d} ; R(s, a)$ is the MDP termination cost; $P_{i}\left(s^{\prime} / s, a\right)$ is the state transition model that computes the probability of the action $a$ resulting in a state transition $s^{\prime} ; D_{i}\left(s^{\prime} / s, a\right)$ is estimated connection cost and $V_{j}\left(s^{\prime}\right)$ is $r_{i} \in$ Cache $_{i}$.

In our experimental model we have replaced IEEE 802.11 by QoS enabled IEEE 802.11e as illustrated in Figure 1.

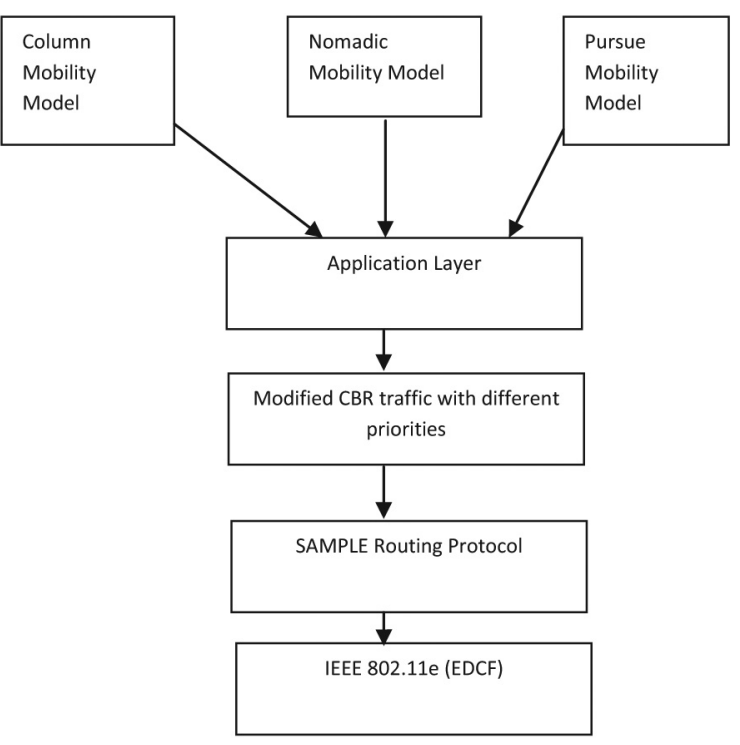

Figure 1. QoS based analysis of SAMPLE routing protocol.

IEEE 802.11e aids more successful packet transmission events by clubbing channel access with priority of packets thus delivering the required QoS services. Also the better channel access aids the packet delivery model of SAMPLE as less number of tries are required for successful transmission.

\section{Group Mobility Models}

Group mobility models have a better performance with less link breakages and they exhibit links which are up for a longer duration [8], [9] and [10]. Thus, in our experimentation we have considered group mobility models like Column Mobility Model (CM), Nomadic Mobility Model (NM) and Pursue Mobility Model (PM). 


\subsection{Column Mobility Model}

A Column Mobility Model [11] models a column of soldiers moving in the forward direction. In this model an initial reference grid is set. Each mobile node is then allowed to move randomly around its reference point. A new reference point is given by Equation 2

$$
N e w_{R P}=\operatorname{Old}_{R P}+A d_{V e c t},
$$

where $\operatorname{Old}_{R P}$ is the mobile nodes previous reference point and $A d_{V e c t}$ the advance vector represents the predefined offset that moves in the reference grid.

\subsection{Nomadic Mobility Model}

The Nomadic Mobility Model (NM) [12] models nomadic societies which move in groups from one location to another. Each group has individual mobile nodes which maintain their own personal spaces and move in random ways. The nomadic mobility model defines a common reference point [13], thus the resulting movements of mobiles nodes is less constrained.

\subsection{Pursue Mobility Model}

The pursue mobility model (PM) [11], [12] attempts to model police officers attempting to catch a criminal. The pursue mobility model follows Equation 3 for each mobile node.

$$
\begin{aligned}
\text { New }_{\text {Pos }}= & \text { Old }_{\text {Pos }}+\text { Acc }^{N}\left(\text { Target }- \text { Old }_{\text {Pos }}\right) \\
& + \text { Rand }_{\text {Vector }}
\end{aligned}
$$

where $A c c^{N}\left(\right.$ Target - Old $\left._{\text {Pos }}\right)$ is the information of the movement of the mobile node being pursued and Rand Vector $_{\text {is }}$ the random offset for each mobile node. The current position of a mobile node, acceleration and random vector are combined to calculate the next position of the mobile node.

\subsection{Performance Analysis of Group Mobility Models}

In this section we discuss the performance analysis of Column, Nomadic and Pursue Mobility models as in [16] to understand their impact on the routing performance of reinforcement learning based routing algorithm SAMPLE.

\subsection{Mobility Performance Analysis Metrics}

We have considered the following mobility metrics from [16] for our analysis of mobility models.

- Average Link Duration: this metric specifies the longest interval of time $[t 1, t 2]$ for nodes $i$ and $j$ forming the link $(i, j)$. This is then averaged for all node pairs for all existing links specifying Equation 4:

$$
L D^{\prime}=\frac{\sum_{t 1=0}^{T} \sum_{i=1}^{N} \sum_{j=i+1}^{N} L D(i, j, t 1)}{P},
$$

where $P$ is no of tuples $(i, j, t 1)$ and $L D(i, j$, $t 1) \neq 0$.

- Average Relative Speed: relative speed is given by Equation 5:

$$
R S T(i, j, t)=|V i(t)-V j(t)|,
$$

where $V i(t)$ and $V j(t)$ is the velocity vector of node $i$ and $j$ at time $t$. The average value of $R S T(i, j, t)$ is given by Equation 6:

$$
R S T^{\prime}=\frac{\sum_{i=1}^{N} \sum_{j=1}^{N} \sum_{t=1}^{T} R S T(i, j, t)}{P},
$$

where $P$ is no of tuples $(i, j, t 1)$ and $R S T^{\prime}(i, j$, $t 1) \neq 0$.

- Average degree of spatial dependence: it is a measure of the extent of similarity of velocities of given two nodes not so far apart, given by $D s(i, j, t)$ and averaged over pair of nodes and time instants and formalized by Equation 7:

$$
D s^{\prime}=\frac{\sum_{t=1}^{T} \sum_{i=1}^{N} \sum_{j=i+1}^{N} D s(i, j, t 1)}{P},
$$

where $P$ is no of tuples $(i, j, t 1)$ and $D s^{\prime}(i, j$, $t 1) \neq 0$.

The performance analysis results of Column Mobility Model (CM), Nomadic Mobility Model 
(NM) and Pursue Mobility Model (PM) are illustrated in Figure 2, Figure 3 and Figure 4.

In Figure 2 it is seen that the average link duration is least for Column Mobility Model. The Pursue Mobility Model exhibits better link duration and outscores Column and Nomadic mo-

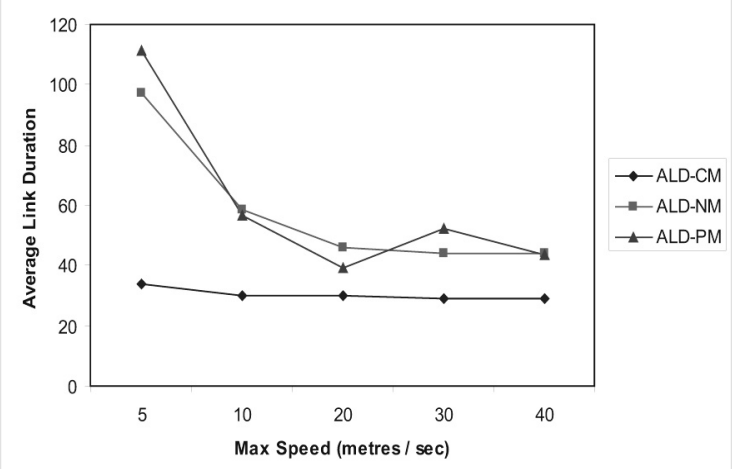

Figure 2. Average link duration for Column, Nomadic and Pursue Mobility models.

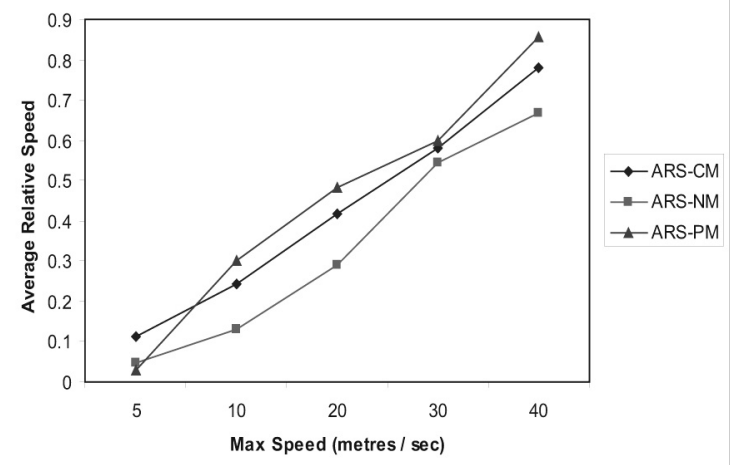

Figure 3. Average relative speed for Column, Nomadic and Pursue Mobility models.

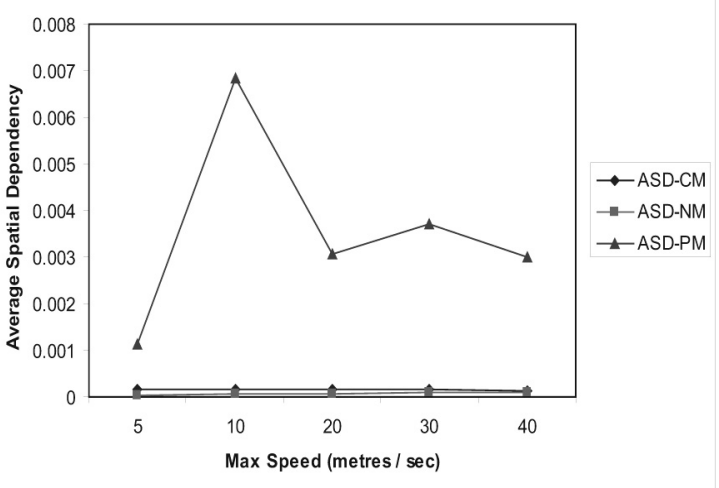

Figure 4. Average spatial dependency for Column, Nomadic and Pursue Mobility models. bility models by $50 \%$ and $4 \%$ respectively. This indicates that nodes in Pursue Model deviate by a small fraction in their groups and exhibit higher link duration.

The average relative speed indicates the mobility pattern of various mobility models. It is observed in Figure 3, that average relative speed is least for Nomadic Model and relatively higher for Column and Pursue Mobility models. The overall values for Column, Nomadic and Pursue Mobility models are least as they are group mobility models and nodes move in synchronized fashion.

In Figure 4, it is seen that the average spatial dependency is highest for Pursue Mobility Model and it outperforms Column and Nomadic Mobility models by $95 \%$ and $98 \%$ respectively. Thus Pursue Mobility Model with higher average link duration and higher spatial dependency performs relatively well as compared to Column and Nomadic Mobility models. These analyses help us to predict the relative performance of SAMPLE a reinforcement learning based routing algorithm on the experimented group mobility models.

\section{Routing Metrics Simulation Environment and Results for QoS Based SAMPLE}

\subsection{Routing Metrics for Performance Evaluation}

- Average latency: is defined as the latency of all packets created at the source divided by the actual application packets received.

- Packet delivery overhead: is defined as the number of transmissions made for every packet in terms of router packets and router broadcast packets, no matter whether that packet is actually delivered or not.

- Average application packet delay: this metric considers the latency of application packets divided by the total application packets sent.

- Total packets dropped: this metric reflects the sum of all packets dropped, no matter whether they may be router generated or application generated packets.

- Packet delivery ratio: this metric defines the ratio between the numbers of packets originated by the application layer to those delivered to the final destination. 


\subsection{Simulation Environment and Results}

NS-2 simulator ver. 2.28 from [17] was used for the study of performance analysis of routing protocol SAMPLE. The underlying MAC Protocol is defined by IEEE 802.11e. Constant bit rate (CBR) traffic sources with different traffic priorities like priority 0 , priority 1 and priority 2. The mobility model used were Column Mobility Model (CM), Nomadic Mobility Model (NM) and Pursue Mobility Model (PM) as provided in the Bonn Motion Tool [18]. The field configurations are $1520 \mathrm{~m} \times 520 \mathrm{~m}$. The traffic generator script called cbrgen.tcl was used to generate CBR scenario of 8 sources at the rate of $4.0 \mathrm{kbps}$ and modified to reflect the corresponding traffic priorities. The number of nodes in the simulation environment was 20 nodes. At least 5 scenarios files for Column, Nomadic and Pursue Mobility models at different maximum speed of 5,10,20, 30 and $40 \mathrm{sec}$ were used for testing protocol like SAMPLE on various QoS based traffic. The simulation based performance analysis results of SAMPLE [2] on QoS based traffic for Column, Nomadic and Pursue Mobility models are illustrated in Figure 5, Figure 6, Figure7, Figure 8 and Figure 9.

In Figure 5 it is shown that the average latency for SAMPLE is on the higher side, for Nomadic Mobility Model especially for lower priority traffic i.e. Priority 1 and Priority 2 traffic as compared to higher priority traffic Priority 0 . It is also observed that the average latency is least for Pursue Mobility Model on all traffic priorities as it is substantiated by better mobility performance metrics.

In Figure 6 it is shown that packet delivery overhead is high for Column and Nomadic Mobility models. For high speed of $40 \mathrm{sec}$, it is observed that Nomadic Mobility model exhibits higher packet delivery overhead as compared to Column and Pursue Mobility Model. The Pursue Mobility Model with its superior mobility metrics exhibits least packet delivery overhead. Also higher mobility of nodes induces larger packet delivery overhead for Column and Nomadic Mobility models.

In Figure 7 it is observed that at lower speed the average application delay is slightly higher for the Pursue Mobility Model at a maximum node speed of $5 \mathrm{sec}$. At higher maximum node speed Pursue Mobility Model performs relatively well as compared to Column and Nomadic Mobility

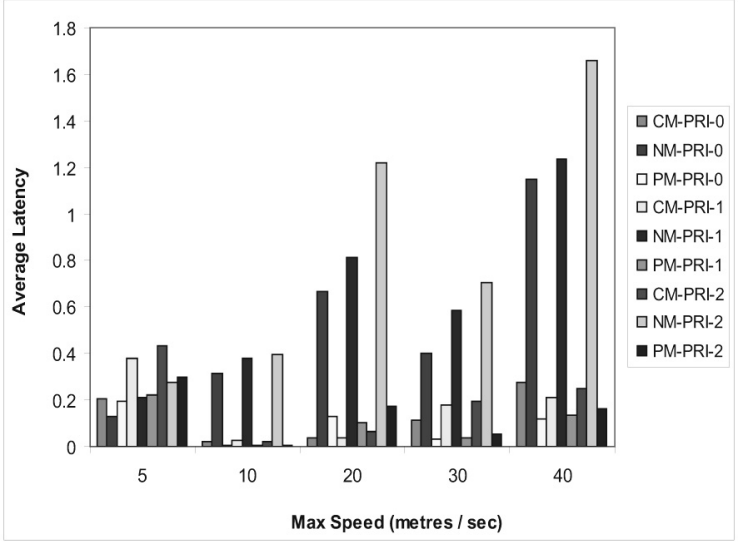

Figure 5. Average latency for SAMPLE with traffic of various priorities.

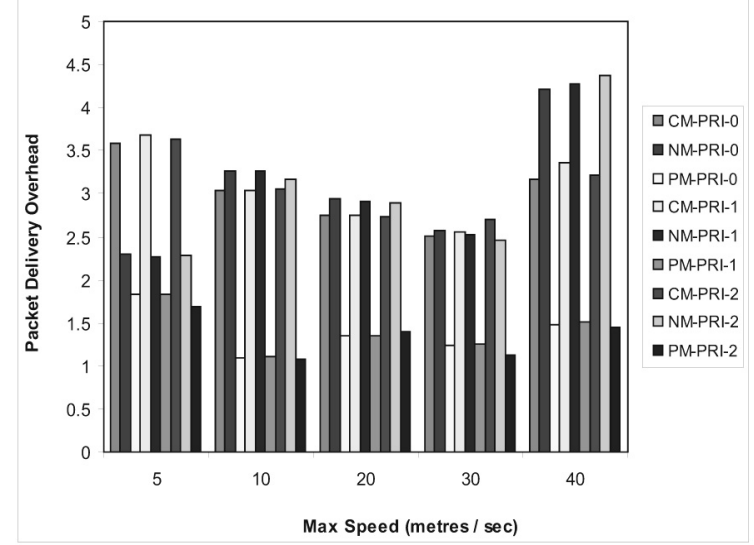

Figure 6. Packet delivery overhead for SAMPLE with traffic of various priorities.

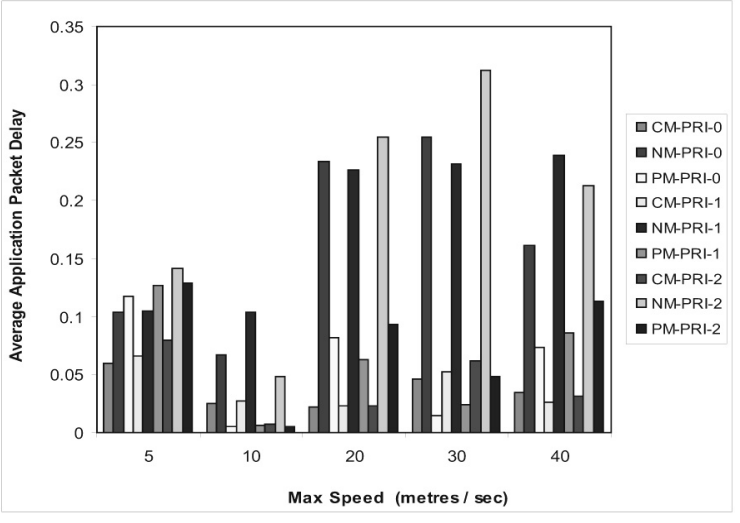

Figure 7. Average application packet delay for SAMPLE with traffic of various priorities.

models. Also, the average application packet delay is least for highest priority traffic Priority 0 as compared to lower priority traffics. 


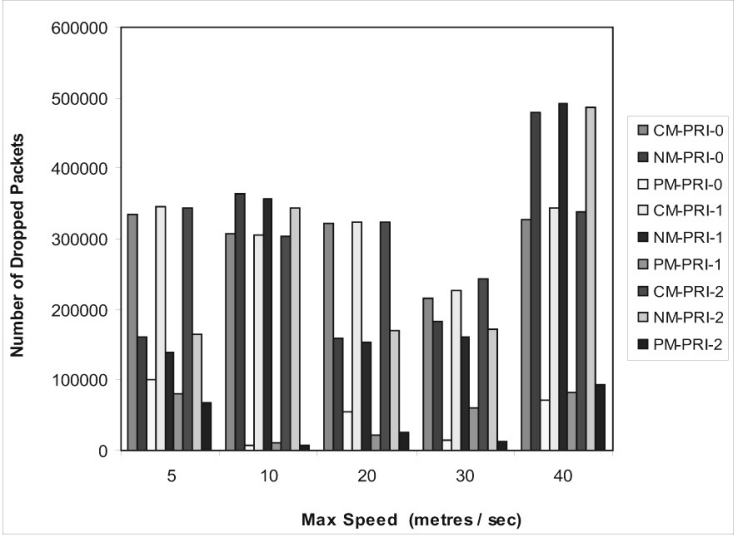

Figure 8. Number of dropped packets of SAMPLE on various traffic priorities.

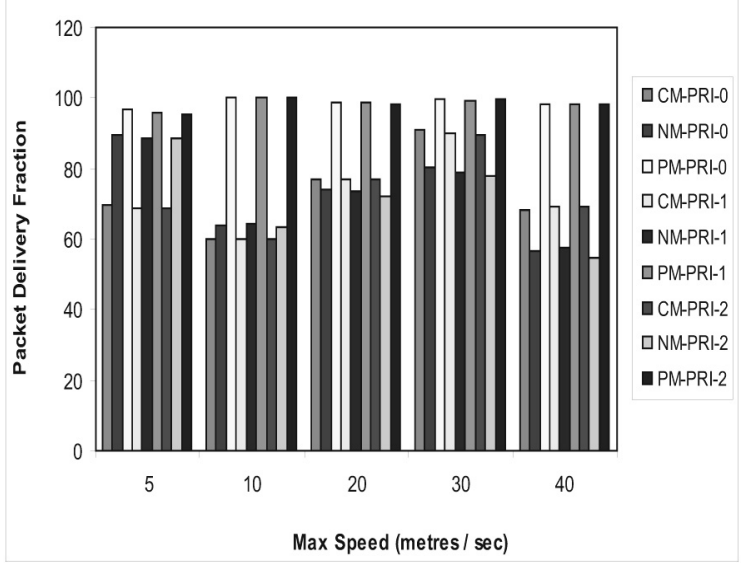

Figure 9. Packet delivery fraction of SAMPLE on various traffic priorities.

In Figure 8, it is observed that the number of dropped packets is higher for SAMPLE on Column Mobility Model and Nomadic Mobility Model. The Pursue Mobility Model has relatively low number of dropped packets because of its superior performance in terms of mobility metrics.

It is observed in Figure 9 that the packet delivery ratio is highest for Pursue Mobility Model for all traffic priority classes. It is seen that for maximum node speed of $40 \mathrm{sec}$, Pursue Mobility Model clearly outscores Column and Nomadic Mobility models by $25.8 \%$ and $27.4 \%$ respectively. This clearly indicates the robustness of the Pursue Mobility Model at high mobility speeds.

\section{Moderate Punishing Algorithm}

In this section we propose a moderate punishing algorithm as a game theoretic modeling of SAMPLE in a temporary non-cooperative environment based on the punishing algorithm proposed [26].

\section{Algorithm: Moderate Punishing Algorithm}

1. Let $N(i)$ be the set of neighbors of node.

2. Every node computes its forwarding policy in a distributed manner using the reinforcement learning algorithm SAMPLE.

3. When a misbehaving neighboring node is detected by a node, the node computes its forwarding policy as follows in Equation 8:

$$
\beta_{i}^{*}=\min \left\{\beta_{i}, \min _{j \in N(i)} \beta_{j}\right\}
$$

where $\beta_{i}$ and $\beta_{j}$ are the reinforcement learning based forwarding policy adapted by node $i$ and the reinforcement learning based forwarding policy available for $j$. $\beta_{i}^{*}$ represents the new policy adapted by node $i$.

4. The network settles down by isolating the misbehaving node and punishing it.

5. The misbehaving node say $n_{i}$ decides to renew cooperation. At this point in time the moderate punishing algorithm will accept its cooperation and the retaliatory punishment of the neighboring nodes of $n_{i}$ and its corresponding negative loop is avoided.

The moderate punishing algorithm is implemented for SAMPLE on the NS-2 simulator for the Pursue Mobility Model and for varying number of non-cooperative nodes. The number of nodes considered for experimentation is 20 . The mobility model employed is Pursue Mobility Model as this model exhibited superior mobility metrics and we have set the maximum speed to $5 \mathrm{sec}$. The numbers of punished nodes which do not cooperate are increased from 1 to 4 for each simulation run lasting $900 \mathrm{sec}$. The MAC layer constitutes IEEE 802.11 and IEEE 802.11e. The traffic constitutes cbr based traffic for SAMPLE on IEEE 802.11 and modified cbr with priority 0 (i.e. the highest priority) for traffic on SAMPLE with IEEE 802.11e. The 
results are illustrated in Figure 10, Figure 11, Figure 12, Figure 13 and Figure 14.

In Figure 10 it is seen that the packet delivery overhead is slightly higher for SAMPLE on IEEE 802.11 as compared to SAMPLE on IEEE $802.11 \mathrm{e}$ for the moderate punishing algorithm. This indicates that SAMPLE on IEEE 802.11 takes more effort to converge to optimal routes due to the presence of nodes which have stopped cooperation for a specified duration.

In Figure 11 it is observed that the number of dropped packets is slightly higher on SAMPLE with IEEE 802.11 as compared with SAMPLE on IEEE 802.11e. Thus, even in the presence of nodes which have stopped cooperation, SAMPLE performs relatively well on IEEE 802.11e and adapts to newer topologies by forgiving nodes which have renewed their decision to cooperate at later stages. The support for QoS traf-

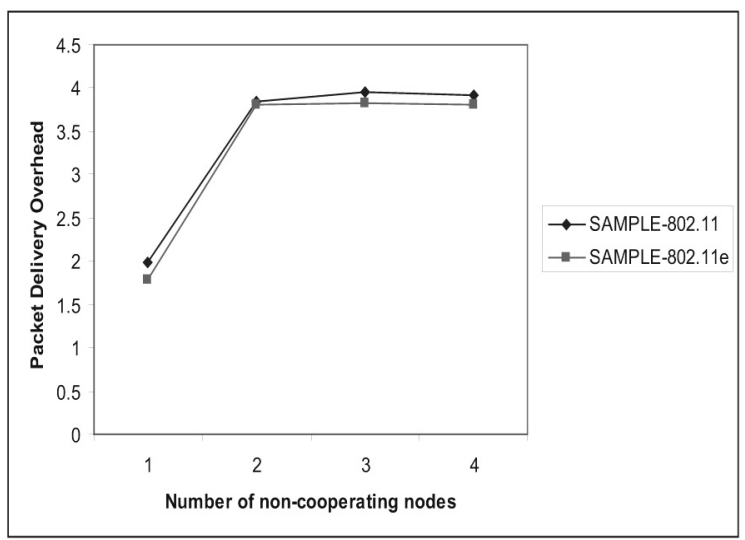

Figure 10. Packet delivery overhead for moderate punishing algorithm for SAMPLE on IEEE 802.11 and IEEE 802.11e.

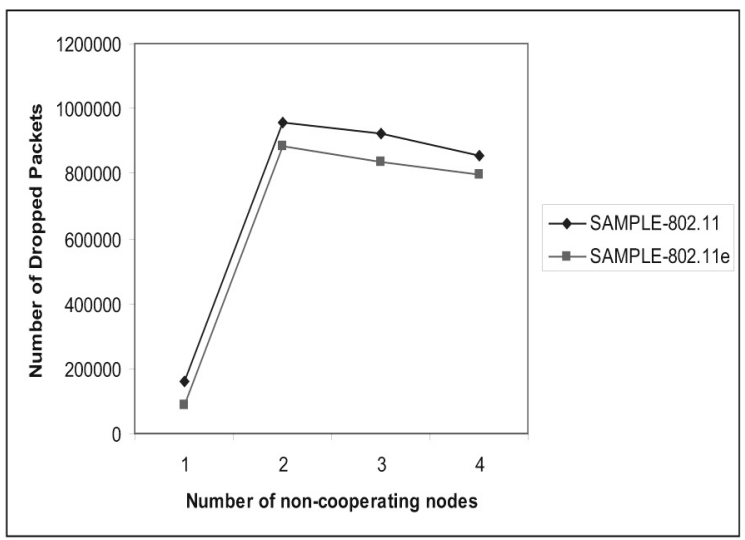

Figure 11. Number of dropped packets for moderate punishing algorithm for SAMPLE on IEEE 802.11 and IEEE 802.11e. fic enables SAMPLE to quickly adapt to newer shortest paths even in the presence of nodes which have stopped cooperation and have been punished with isolation for a specific duration.

In Figure 12 it is observed that for the implementation of moderate punishing algorithm for SAMPLE, the average latency is initially higher for SAMPLE on $802.11 \mathrm{e}$, but as the simulation progresses and nodes renew or stop their cooperation, SAMPLE on IEEE 802.11e continues to perform well as compared to SAMPLE on IEEE 802.11e.

In Figure 13 it is seen that SAMPLE on IEEE 802.11e clearlyoutperforms SAMPLE on IEEE 802.11 in terms of average application packet delay. Thus IEEE 802.11e with better mechanism to support QoS enabled traffic supports faster dissemination of application packets.

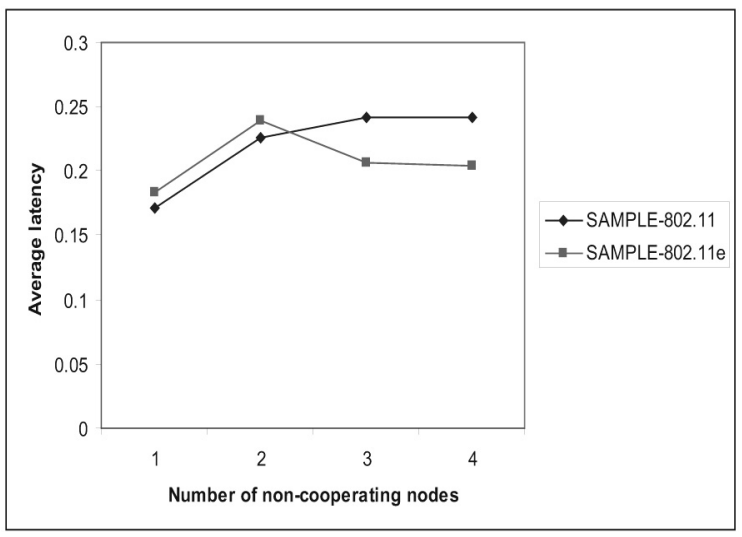

Figure 12. Average latency for moderate punishing algorithm for SAMPLE on IEEE 802.11 and IEEE $802.11 \mathrm{e}$.

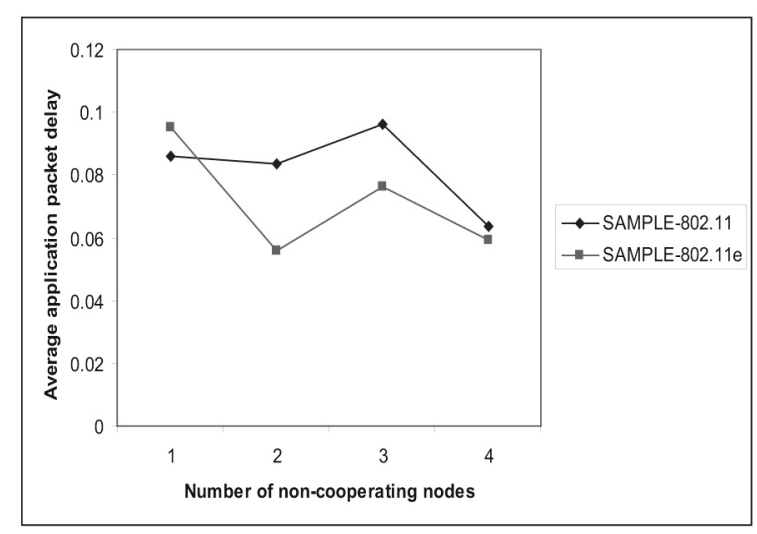

Figure 13. Average application packet delay for moderate punishing algorithm for SAMPLE on IEEE 802.11 and IEEE 802.11e. 


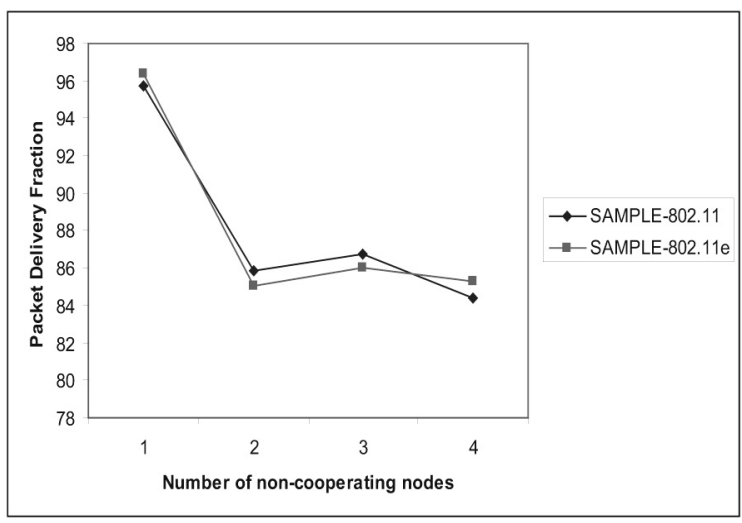

Figure 14. Packet delivery fraction for moderate punishing algorithm for SAMPLE on IEEE 802.11 and IEEE 802.11e.

In Figure 14 it is observed that the packet delivery fraction is more or less the same for SAMPLE on IEEE 802.11 and IEEE 802.11e. Along with the increased number of nodes which stop or renew their cooperation, it is observed that SAMPLE on IEEE 802.11e exhibits more stable performances.

\section{Conclusion}

Routing algorithms form the backbone of adhoc networks. Providing Quality of service (QoS) with different traffic priorities is an important problem on such networks. QoS is not just the function of one layer; it can be visualized across the protocol stack. Thus at network layer we consider SAMPLE which has superior performance in terms of congestion capabilities for high load traffic. At the data link layer we consider IEEE 802.11e which has proven support for QoS as compared to IEEE 802.11. We also consider group mobility models, which due to their group movements have superior mobility metrics as compared to other models. Our experiments observed the superior performance of Pursue Mobility Model. The Pursue Mobility Model exhibited better link duration and outscored Column and Nomadic mobility models by $50 \%$ and $4 \%$ respectively. Also, the average spatial dependency was the highest for Pursue Mobility Model and it outperformed Column and Nomadic Mobility models by $95 \%$ and $98 \%$ respectively. SAMPLE on Pursue Mobility Model had better performance for various routing and QoS metrics on IEEE 802.11e. The operation of SAMPLE on Pursue Mobility Model has relatively low number of dropped packets because of its superior performance in terms of mobility metrics. The packet delivery ratio of SAMPLE is the highest for Pursue Mobility Model for all traffic priority classes. It is observed that for maximum node speed of $40 \mathrm{sec}$, Pursue Mobility Model clearly outscores Column and Nomadic Mobility models by $25.8 \%$ and $27.4 \%$ respectively. Adhoc networks are constrained by resources like bandwidth and energy. Thus we propose a moderately punishing algorithm for modeling the behavior of routing algorithm SAMPLE with resource constraints on IEEE 802.11e. Along with the increased number of nodes which stop or renew their cooperation, it is observed that SAMPLE on IEEE 802.11e exhibits more stable performances in terms of packet delivery ratio. In the future, we would like to extend our QoS study for video based traffic on MANET.

\section{Acknowledgments}

The authors would like to acknowledge the support of Computer Society of India for the research work through a Mini Research Grant (File No. 1-14/2012-06).

\section{References}

[1] S. Weitholter, C. HoENE, Design and Verification of an IEEE 802.11e EDCF Simulation Model in NS 2.26, TKN Technical Report Series, Berlin, (2003).

[2] J. Dowling, E. Curran, R. Cunningham, V. CAHILL, Using Feedback in Collaborative Reinforcement Learning to Adaptively Optimize MANET Routing, IEEE Transactions on Systems, Man and Cybernetics - Part A: Systems and Humans, Vol. 35, No. 3, pp. 360-375, (2005).

[3] J. Yoon, M. LiU, B. Noble, Random Waypoint Considered Harmful, Proceedings of IEEE INFOCOM 2003, San Francisco, CA, (2003).

[4] S. Sarkar, T. G. Basavaraju, C. Puttamadappa, Ad Hoc Mobile Wireless Networks, Principles, Protocols and Applications, Auerbach Publications, pp. 267-273, (2008).

[5] E. D. Puschita, T. P. Palade, QoS Perspectives for Wireless Scenarios, In the Proceedings of BroadBand Europe, (2005).

[6] E. Puscita, T. Palade, L. Chitra, Performance Evaluation of DF Vs EDCF Data Link Layer Access Mechanisms for Wireless LAN Scenarios QoS Perspective, Proceedings of 7 th International Conferences on Telecommunication in Modernization Cable and Broadcasting Services, September 2830, (2005). 
[7] A. W. Moore, C. G. AtKenson, Prioritized Sweeping: Reinforcement Learning with Less data and Less Time, Machine Learning, Vol. 13, (1993).

[8] S. A. Kulkarni, G. R. RaO, Applying Optimized Mobility Model for Reinforcement Learning Based Routing Algorithm for Mobile Ad-Hoc Networks, Journal of Computer Network and Internet Research, Vol. 10, No. 1, pp. 49-57, (2010).

[9] X. Hong, M. Gerla, G. Pai, C-C. Chiang, A Group Mobility Model for Ad Hoc Wireless Networks, ACM/IEEE International Symposium on Modeling Analysis and Simulation of Wireless and Mobile Systems (MSWIM), Seattle, WA, USA, (1999).

[10] S. A. Kulkarni, G. R. RaO, Mobility and EnergyBased Performance of Temporally Ordered Routing Algorithm for Ad Hoc Wireless Network, IETE Journal of Technical Review, Vol. 25, No. 4, pp. 222-227, (2008).

[11] M. SANChEZ, P. MANZONI, A java-based ad hoc network simulator, Future Generation Computer Systems, Vol. 17, No. 5, pp. 573-583, (2001).

[12] M. SANCHEZ, Mobility models, http://www . disca.upv.es/misan/mobmodel.htm

[13] T. CAMP, J. Boleng, V. DaviES, Survey of Mobility Models, http://toilers.mines.edu/papers/ psgz/models.ps.gz

[14] C. T. Calafate, M. P. Malumbers, J. Oliver, J. C. CANO AND P. MANZONI, QoS Support in MANETs: A Modular Architecture Based on the IEEE 802.11e Technology, IEEE Transactions on Circuits and Systems for video technology, Vol. 19, No. 5, pp. 678-692, (2009).

[15] C. T. Calfate, P. Manzoni, M. P. Malumbres, On the Interaction Between IEEE 802.11e and routing protocols in Mobile Ad-Hoc Networks, In the Proceedings of 13th Euromicro Conference on Parallel Distributed and Network-based Processing (PDP), Lugano, Switzerland, (2005).

[16] F. BAI, N. SAdagopan, A Helmy, The IMPORTANT framework for analyzing the Impact of Mobility on Performance of RouTing protocols for Adhoc NeTworks, Elsevier Ad Hoc Networks, 1, pp. 383-403, (2003).

[17] The Network Simulator - NS-2, http://www.isi.edu/nsam/ns/

[18] Bonn Motion v 1.5a - A mobility scenario generation and analysis tool, http://net.cs.unibonn.de/wg/cs/applications/bonnmotion/

[19] C. E. Perkins, E. M. Belding-Royer, Quality of Service for Ad-Hoc On-Demand Distance Vector Routing, Internet draft, (2001), http://tools. ietf.org/html/draft-perkins-manetaodvqos -00

[20] V. Loscrì, F. D. RANGO, S. MARANO, Performance Evaluation of On-Demand Multipath Distance Vector Routing Protocol over two MAC layers in Mobile Ad Hoc Networks, In Proceedings of ICT, (2004).
[21] L. KhoukHI, S. CherkaOuI, Intelligent QoS management for multimedia services support in wireless mobile ad hoc networks, Computer Networks 54, pp. 1692-1706, (2010).

[22] A. Munaretto, M. Fonseca, Routing and quality of service support for mobile ad hoc networks, Computer Networks 51, pp. 3142-3156, (2007).

[23] T. BheEMarjuna Reddy, I. Karthigeyan, B. S. MANOJ, C. SIVA RAM MURTHY, Quality of service provisioning in ad hoc wireless networks: a survey of issues and solutions, Ad Hoc Networks 4, pp. 83-124, (2006).

[24] M. A. Igartua, L. J. D L CLlopis, V. C. Frías, E. S. GARGALlO, A game-theoretic multipath routing for video-streaming services over Mobile Ad Hoc Networks, Computer Networks 55, pp. 2985-3000, (2011).

[25] D. LEVIN, Punishment in Selfish Wireless Networks A Game Theoretic Analysis, In Proceedings of First Workshop on the Economics of Networked Systems (NetEcon06), (2006).

[26] E. Altman, K. A. Arzad, P. Michiardi, R. Molva, Non cooperative forwarding in ad hoc networks, In Proceedings of IFIP Networking, (2005).

Received: July, 2012 Revised: November, 2012 Accepted: January, 2013

Contact addresses: Dr. Shrirang Ambaji Kulkarni, Associate Professor Department of Computer Science and Engineering Gogte Institute of Technology Belgaum - 590008, Karnataka, India e-mail: sakulkarni@git.edu

Dr. G. Raghavendra Rao, Professor Department of Computer Science and Engineering National Institute of Engineering Mysore-570008, Karnataka, India e-mail: grrao56@gmail.com

SHRIRANG AMBAJI KULKARNI obtained his B.E. in Computer Science and Engineering from Karnataka University Dharwad, in the year 2000 and M. Tech. in Computer Network Engineering from Visvesvaraya Technological University India, in the year 2004. He obtained his Ph.D. in the faculty of Computer and Information Science from Visvesvaraya Technological University India in the year 2012. He is currently working as Associate Professor, in the Department of Computer Science and Engineering, Gogte Institute of Technology, Belgaum India. He has published more than 20 papers in international and national journals and conferences. His current research interest includes reinforcement learning algorithms, wireless ad-hoc networks, cyber security. He is a member of ACM, CSI and ISTE.

G. RAGHAVENDRA RAO obtained his Masters in Engineering from Indian Institute of Science Bangalore India in the year 1987 and his Ph.D. from the University of Mysore in the year 1999. He is currently working as Professor in the Department of Computer Science and Engineering at the National Institute of Engineering Mysore, India. He has published more than 40 papers in international and national journals and conferences. His current research interest includes genetic algorithms, wireless networks, cryptography and network security. He is a member of IEEE, ACM and ISTE. 